\title{
The retroperitoneum
}

\section{Francisco Llabrés-Díaz}

\section{Normal retroperitoneum}

The retroperitoneum is an extraperitoneal space situated ventral to the vertebrae and paraspinal muscles; it extends along the length of the entire abdomen from the diaphragm to the anus. The lateral boundaries are the abdominal and pelvic walls. The ventral boundary is the dorsal parietal peritoneum. Therefore, conditions affecting the peritoneum may spare the retroperitoneum.

Organs that project freely into the abdominal, pelvic or scrotal cavities with an almost complete covering of peritoneum are considered to be intraperitoneal. However, those organs situated close to the walls of the abdominal or pelvic cavities and covered on one surface only by peritoneum are considered to be retroperitoneal. Some of these organs are too small to be seen, whilst others are commonly seen on radiographs because they are surrounded by fat (Figure 5.1). The retroperitoneal organs include:

- Kidneys

- Ureters

- Adrenal glands

- Aorta

- Caudal vena cava

- Coeliac and cranial mesenteric arteries, renal and deep circumflex vessels

- Lymph nodes:

- The lumbar lymphocentre: lumbar aortic and renal lymph nodes

- The iliosacral lymphocentre: medial iliac, hypogastric and sacral lymph nodes.

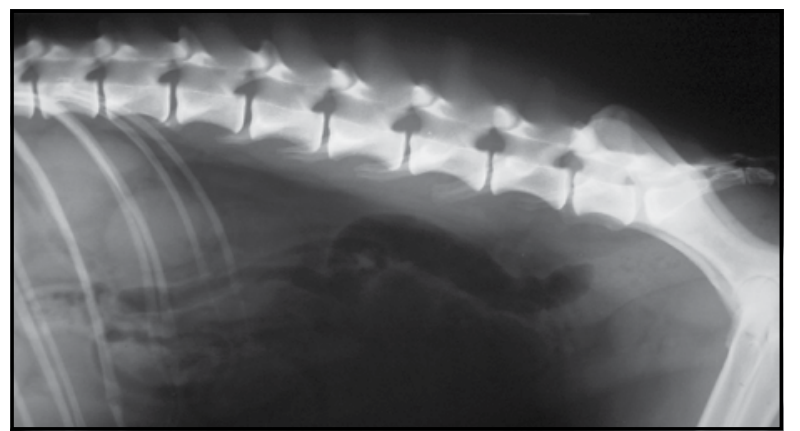

5.1 Lateral abdominal radiograph showing the normal retroperitoneum of a 6-year-old male neutered Whippet. The presence of fat allows identification of some of the retroperitoneal structures, especially the left kidney.
- Cisterna chyli and lymphatic ducts

- Neural structures (including sympathetic nerves and ganglia)

- Loose connective tissue.

\section{Connection with other spaces in the body}

The loose connective tissue mesh that fills the retroperitoneum does not provide a complete barrier to disease and therefore rapid spread of pathology can occur. In addition, the retroperitoneum communicates cranially with the mediastinum (Figure 5.2) and

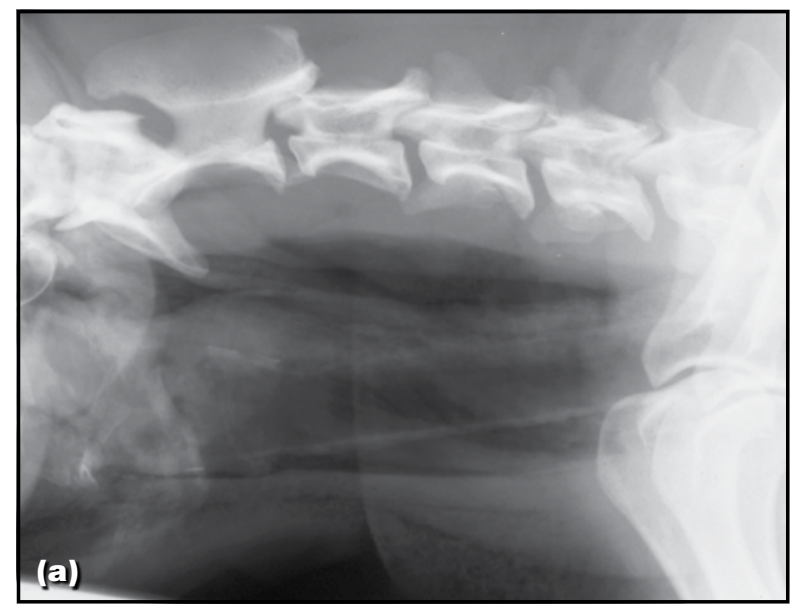

(b)

5.2 Radiographs of an 11-year-old male neutered English Springer Spaniel that had suffered iatrogenic damage to the upper respiratory tract. (a) Lateral radiograph of the cervical area. Air can be seen tracking along the fascial planes. (b) Lateral thoracic radiograph. The clear boundaries of the mediastinal structures indicate the presence of pneumomediastinum. (continues) 


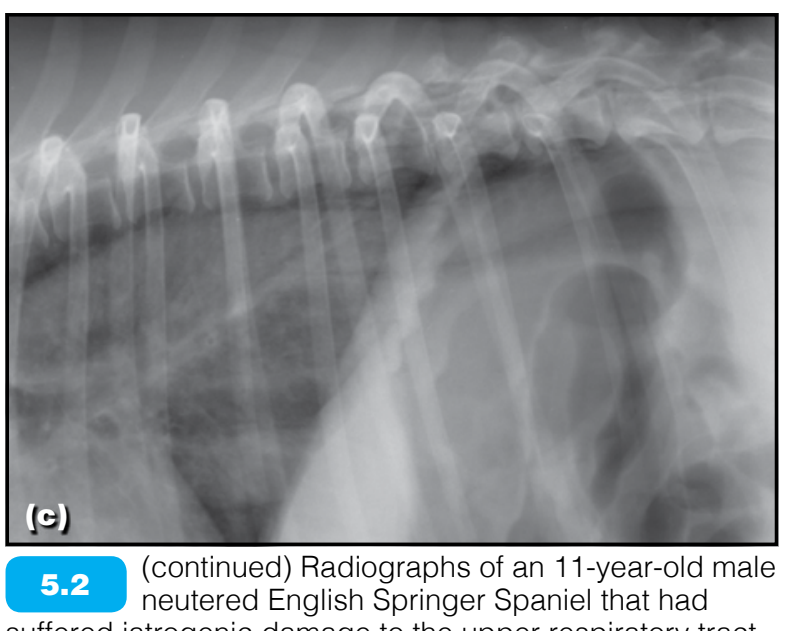

suffered iatrogenic damage to the upper respiratory tract. (c) Close-up of a lateral radiograph of the thoracolumbar region. The aorta is clearly seen owing to the presence of air that is extending from the caudal mediastinum into the retroperitoneum.

caudally with the pelvic canal, reaching the anus. This connection is of great importance from a clinical perspective and the presence of radiographic signs of retroperitoneal disease should prompt the investigation of these neighbouring spaces to confirm or rule out the presence of pathology and to investigate the origin of the disease process.

\section{Ultrasonography}

The relative indications and advantages of ultrasonography are covered in Chapter 3. Particular considerations related to the retroperitoneum are:

- Ultrasonography is particularly helpful in cases where radiographs have identified an increase in retroperitoneal radiopacity, as it allows determination of the internal architecture (fluid versus tissue) and vascularity of the lesion

- Ultrasonography is important for selecting areas to aspirate or biopsy and is extremely useful for obtaining ultrasound-guided samples

- It can be difficult to determine accurately the true extent of diffuse pathology and very large masses with ultrasonography, in comparison with radiographs or advanced imaging techniques. A multi-technique approach is better in this scenario.

\section{Technique}

Several ultrasonographic windows to the retroperitoneum are available. Selection is mostly based on the ultrasonographer's personal preference. The author favours a lateral approach to this area with the animal in lateral recumbency. The ventral aspect of the vertebral bodies is easily identified by the presence of acoustic shadowing and indicates the dorsal boundary of the retroperitoneum. A combination of longitudinal and transverse planes with respect to the long axis of the body will enable examination of the entire retroperitoneum. Following the aorta and caudal vena cava in a caudocranial direction is useful.
The normal ultrasonographic appearance of the retroperitoneal organs is covered in the relevant Chapters. The general appearance of the retroperitoneal space, however, is a loose array of echogenicities (Figure 5.3), similar to that of the omentum within the peritoneal cavity. Fluid is not usually apparent in the normal retroperitoneum.
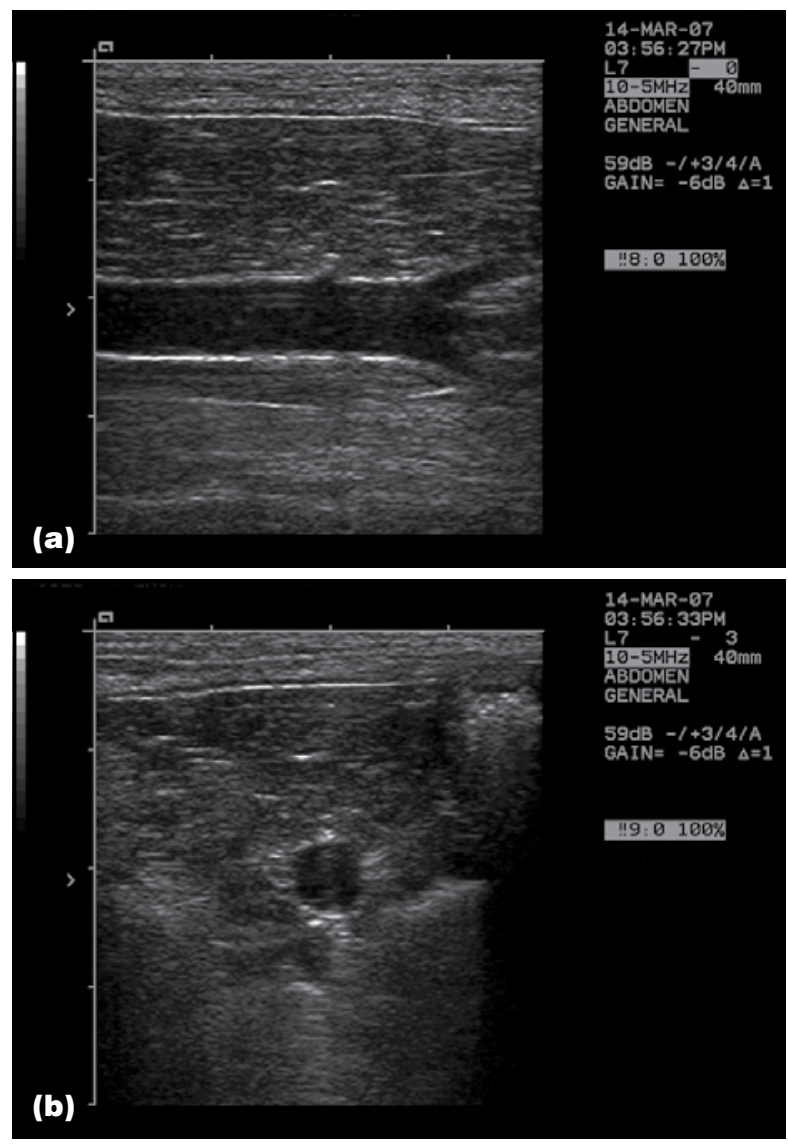

5.3 (a) Ultrasonogram of the normal retroperitoneum of a 13-year-old neutered Yorkshire Terrier bitch obtained in a longitudinal plane, parallel to the long axis of the body. Cranial is to the left. The aorta can be seen in the mid-field, surrounded by the normal retroperitoneum. (b) Transverse image of the same area. Dorsal is to the left of the image. The shadowing caused by the descending colon is visible to the right of the image.

\section{Overview of additional imaging modalities}

Extraordinary anatomical detail, free of superimposed neighbouring structures, can be obtained with computed tomography (CT) and magnetic resonance imaging (MRI). This makes them very useful in cases of diffuse retroperitoneal pathology, especially when surgery is contemplated.

Financial constraints, the limited availability of $\mathrm{CT}$ and $\mathrm{MRI}$ in some areas and the difficulty in accurately interpreting the resulting images constitute the main drawbacks. CT is quicker than $\mathrm{MRI}$ and is generally less affected by movement artefacts. A complete MRI examination of patients with diffuse pathology extending into other 
anatomical areas, such as the mediastinum, can be very lengthy and frustrating. CT is the advanced imaging technique of choice in these cases.

\section{Technique}

A detailed discussion of $\mathrm{MRI}$ and $\mathrm{CT}$ techniques, including the different sequences (MRI) or windows (CT) available to the radiologist, is beyond the scope of this Chapter, thus only the general concepts are mentioned here.

\section{Magnetic resonance imaging}

- As a rule of thumb, STIR images, T2-weighted images and equivalent pre- and post-contrast (using intravenously injected paramagnetic contrast medium) enhanced T1-weighted images should be obtained when investigating diffuse retroperitoneal pathology. These facilitate the detection of pathological and/or contrast enhancing areas, respectively, which appear hyperintense compared with the surroundings.

- Pathology and normal accumulations of fluid appear hyperintense to the surroundings on STIR and T2-weighted images. Fat is also of high signal intensity on T2-weighted images but not on STIR, which is why STIR images are helpful.

- Fat is of high signal intensity on T1-weighted images, whereas fluid is of low signal intensity. Contrast enhanced areas are brighter than on pre-contrast T1-weighted images.

- The array of sequences available to the radiologist and the true multiplanar capabilities of $\mathrm{MRI}$ can make this a superior technique to $\mathrm{CT}$, especially if severe soft tissue or vertebral canal disease is present.

\section{Computed tomography}

Images from bone and soft tissue windows should be obtained before and after injection of contrast medium (intravenous, iodinated, water-soluble), plus post-processing reconstruction planes, software permitting.

\section{Retroperitoneal diseases}

\section{Fluid}

Fluid accumulation within the retroperitoneum results in a loss of contrast on plain radiographs, because the soft tissue structures within the retroperitoneum are no longer highlighted by the surrounding fat. A small volume of fluid results in streaking or mottling of the retroperitoneal area. Larger volumes of fluid obscure all retroperitoneal detail (Figure 5.4) and can result in expansion of the retroperitoneal space with ventral deviation of the colon and small intestines.

Blood is radiographically indistinguishable from urine or other fluids, but this differentiation may prove critical for the patient. For example, a traumatic haematoma usually resolves over time, and indeed there is the risk of further haemorrhage if a haematoma is disturbed during exploratory surgery. However, the presence of urine warrants intervention to prevent tissue necrosis and systemic illness.

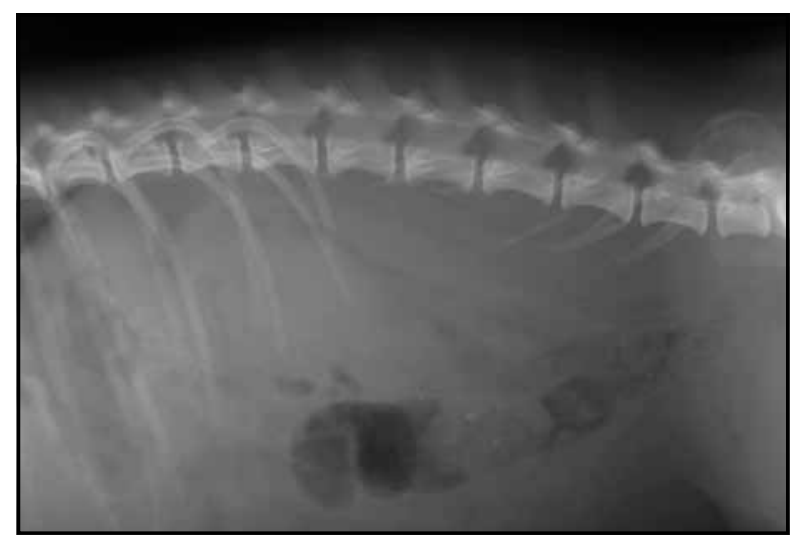

5.4 Lateral abdominal radiograph of a 4-year-old neutered Jack Russell Terrier bitch following a recent road traffic accident. There is retroperitoneal enlargement and loss of detail due to fluid accumulation. Fractures of several vertebral transverse processes are evident and marked swelling of this area also contributes to the retroperitoneal enlargement.

Both radiography and ultrasonography are helpful in determining whether retroperitoneal disease is present. However, ultrasonography is more sensitive than radiography in the detection of a small amount of fluid (Figure 5.5).

- A moderate amount of fluid causes a striated or marble-like appearance due to a combination of areas of fluid and relatively hyperechoic fatty tissue. A little free fluid may accumulate adjacent to the organ of origin; for example, perirenal free fluid is a common sequel to acute renal failure.

- A large accumulation of fluid will be easily detectable on ultrasonography as an anechoic area with variable small internal echogenicities (Figure 5.6), resulting from the cellularity and other characteristics of the fluid.

- Carcinomatosis involving the retroperitoneum is often associated with the presence of fluid, resulting in a striated or marble-like pattern (see above) (Figure 5.7). There may also be an

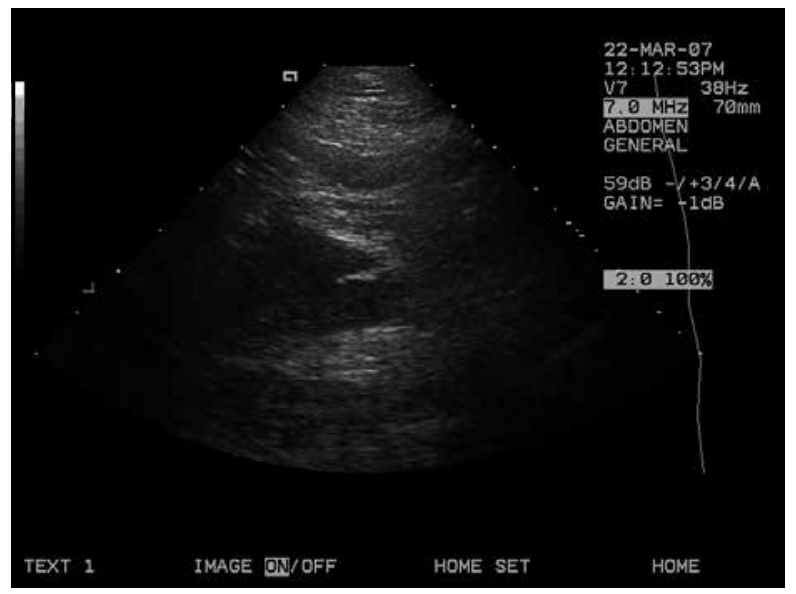

5.5 Ultrasonogram of the retroperitoneum of a 5-year-old male neutered English Springer Spaniel presented with pyrexia and abdominal pain. A small anechoic pocket of fluid was detected caudal to the renal area. A migrating foreign body was suspected but was not found. 


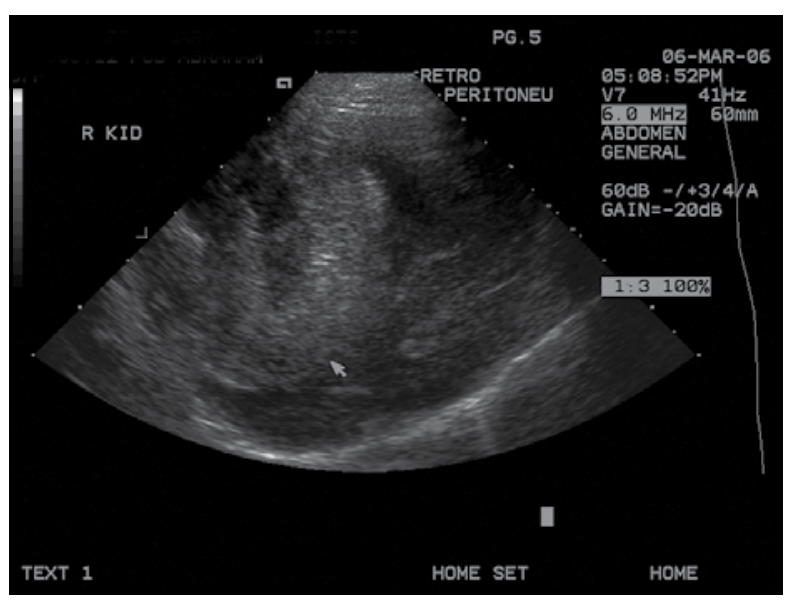

5.6 Ultrasonogram of the cranial retroperitoneum of 5.6 the dog shown in Figure 5.4. There is fluid accumulation around the right kidney. Cranial is to the left.
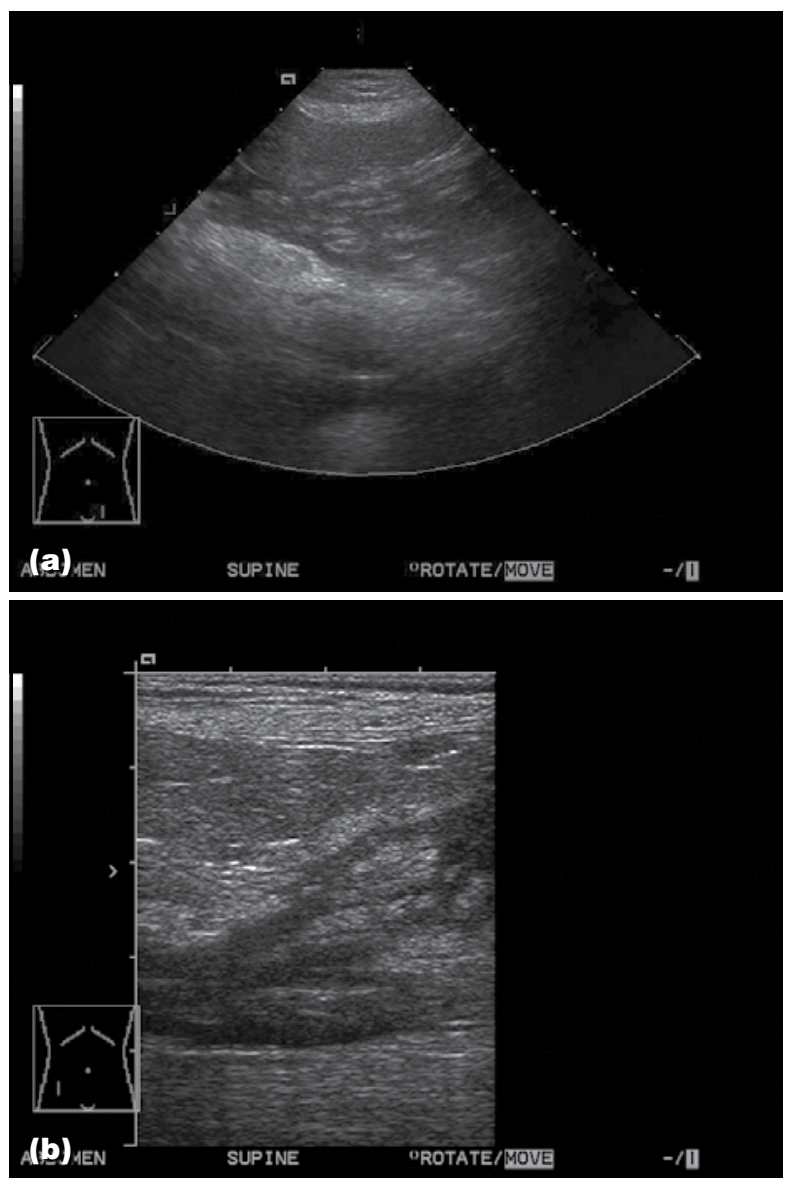

5.7 Ultrasonograms of the central retroperitoneum of a 9-year-old male neutered Shih Tzu cross obtained with (a) a $7 \mathrm{MHz}$ curvilinear transducer and (b) a $10 \mathrm{MHz}$ linear transducer. Cranial is to the left. This pattern is consistent with fluid accumulation and carcinomatosis but is not specific for this condition. The final diagnosis was disseminated caudal retroperitoneal mast cell tumour.

increase in echogenicity of the soft tissues, but a certain level of experience is needed before subtle retroperitoneal changes in echogenicity can be detected confidently.
Ultrasound-guided aspiration will allow determination of the type of fluid present, and thus the best course of action.

\section{Fluid of renal or ureteric origin}

- Acute renal failure:

- This is a non-specific finding with many causes, including obstructive uropathy, toxicity, infection or neoplasia

- The volume of fluid is usually small and it accumulates in contact with the cranial or caudal capsule of the affected kidney(s)

- This finding may be unilateral or bilateral, depending on the insult. Systemic injuries, such as intoxication or haematogenous infection, are usually bilateral

- Ureteral obstruction or pyelonephritis may be unilateral. In cases of unilateral fluid there may be no concurrent signs of renal failure noted on serology or urinalysis.

- Retroperitoneal fluid can also be seen in association with a traumatic episode and secondary leakage of urine:

- If the fluid becomes encapsulated, the lesion is defined as a urinoma

- Uroabdomen is diagnosed when the creatinine and potassium levels in the free fluid are higher than those in serum

- Extravasation of sterile urine can be asymptomatic for 1-2 days. Clinical signs will develop sooner if the extravasated urine is infected

- A urogenital contrast study (Figure 5.8) may be needed to determine the origin of the leakage into the retroperitoneum before surgery is performed

- The presence of other lesions secondary to the traumatic episode (e.g. bladder or abdominal wall rupture, pneumothorax, rib fractures or fractures elsewhere, lung contusion, diaphragmatic hernia) should be investigated.

- Ultrasonography should be used to confirm that a retroperitoneal accumulation of fluid exists, assess concurrent lesions and to obtain an ultrasound-guided sample to characterize the nature of the fluid.

\section{Haemorrhage}

Possible causes of haemorrhage include:

- Trauma

- Rupture of highly vascularized masses

- Vascular rupture:

- Neoplastic infiltration

- Penetrating injuries

- Vascular anomalies.

- Coagulopathies:

- Disseminated intravascular coagulation (DIC)

- Rodenticide toxicity

- Immune-mediated thrombocytopenia. 

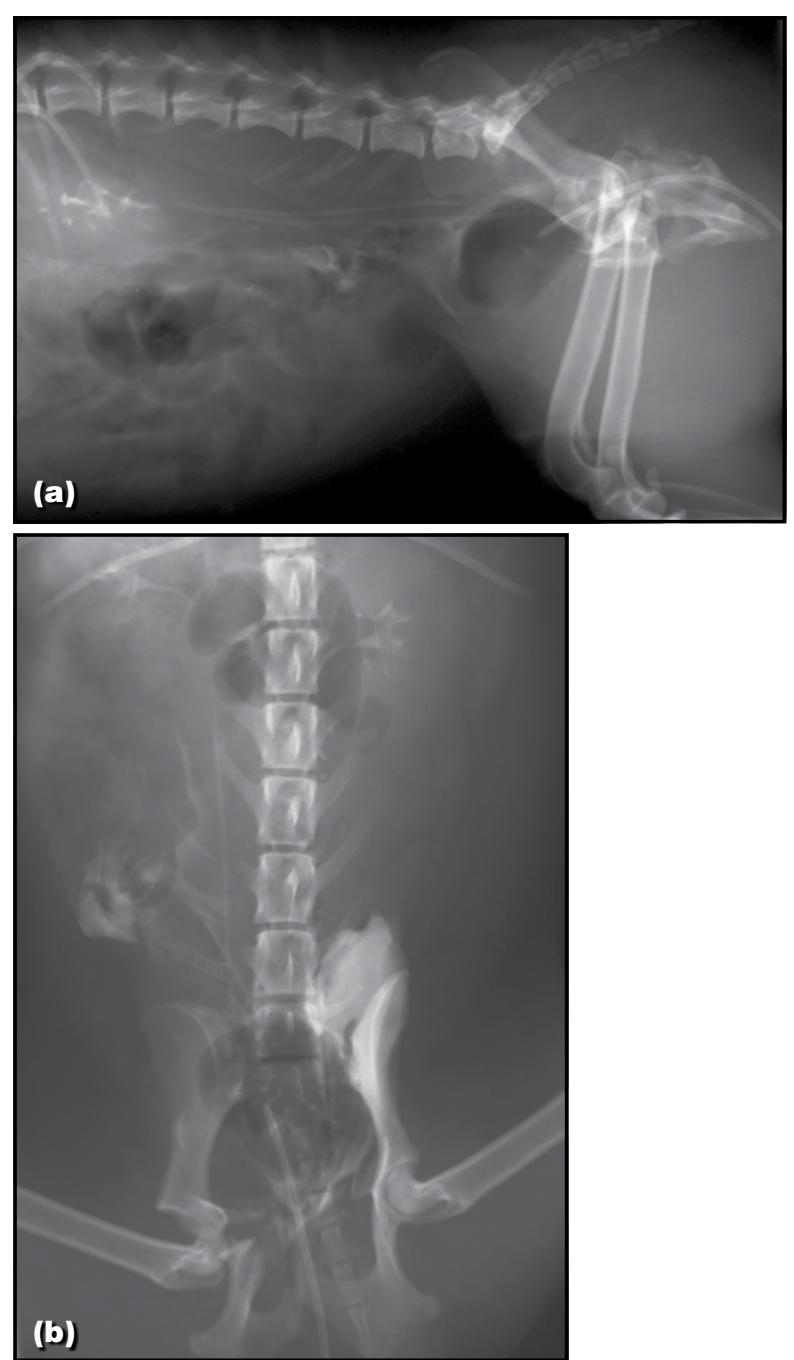

5.8 (a) Lateral and (b) VD radiographs of the dog in Figure 5.4 after bladder catheterization, air insufflation and intravenous urography. The presence of positive contrast medium outside the bladder neck, extending cranially and surrounding intestinal loops in places confirms the suspicion of uroretroperitoneum and uroperitoneum.

In cases of trauma, it is important to determine whether other areas of the body are affected and, if possible, to ascertain the origin of the bleeding. The spleen, liver and kidneys (in that order) are the most common abdominal sources of haemorrhage; ultrasonography is useful in determining the origin of the haemorrhage. Adrenal gland phaeochromocytomas are the particular neoplasm associated with retroperitoneal haemorrhage. The haemorrhage may originate from rupture of the neoplasm or invasion or thrombosis of neighbouring vessels. Haemoperitoneum may coexist with haemorrhage in the retroperitoneum. Ultrasonography is particularly helpful in this scenario and findings may include:

- Abnormal adrenal gland(s)

- Retroperitoneal fluid

- Detection of metastases.

The presence of hypertension should raise the suspicion of a phaeochromocytoma.
Cullen's sign (periumbilical discoloration or ecchymosis), together with haemoglobinuria, has been reported in a dog with a large retroperitoneal haemorrhage. Retroperitoneal or peritoneal haemorrhage should be ruled out when this infrequent sign is detected. Focal renal subcapsular haematomas may be seen following blunt trauma and this area needs to be specifically assessed using ultrasonography. Advanced imaging may provide more detailed information about damage to skeletal structures or may verify the presence of a mass lesion.

\section{Foreign bodies}

Migrating foreign bodies are probably the most common cause of chronic and recurrent cases of retroperitonitis. Other causes include penetrating injuries, extension of local infections, such as severe pyelonephritis, and iatrogenic damage to the urethra. Retroperitoneal infections are uncommonly associated with reactions to ligatures used in neutering surgeries.

Appropriate therapy and prognosis are determined by the underlying cause and whether the process is chronic or acute. Chronic cases are likely to be associated with fever, pain, fluctuating flank abscesses and discharging sinuses. Back pain and neurological deficits are also possible. In these cases, local drainage and systemic antibiotic therapy are likely to resolve the problem only partially, and recurrence is very likely.

Migrating foreign bodies may originate from the oral cavity or sites more caudal in the respiratory or gastrointestinal systems.

- Migrating plant awns may gain access to the cranial retroperitoneum through the oesophageal wall at the sharp point of the oesophageal flexure just cranial to the cardia.

- Alternatively, access to the retroperitoneum is gained by migrating through the dorsal attachments of the diaphragm from the caudodorsal thoracic cavity after inhalation and migration out of the lung. The presence of a cough and transient radiographic changes in the lungs before the development of changes in the lumbar spine supports this hypothesis in some patients.

\section{Radiographic findings}

- Normal radiographs do not rule out retroperitoneal extension of the infection during the acute phase of the disease.

- Some or all of the following radiographic patterns can be identified in these cases:

- Diffuse increase in opacity with a large volume of free fluid or a mass

- Focal soft tissue opacities, possibly with internal gas opacities, if there is abscessation or an open discharging sinus (Figure 5.9) 

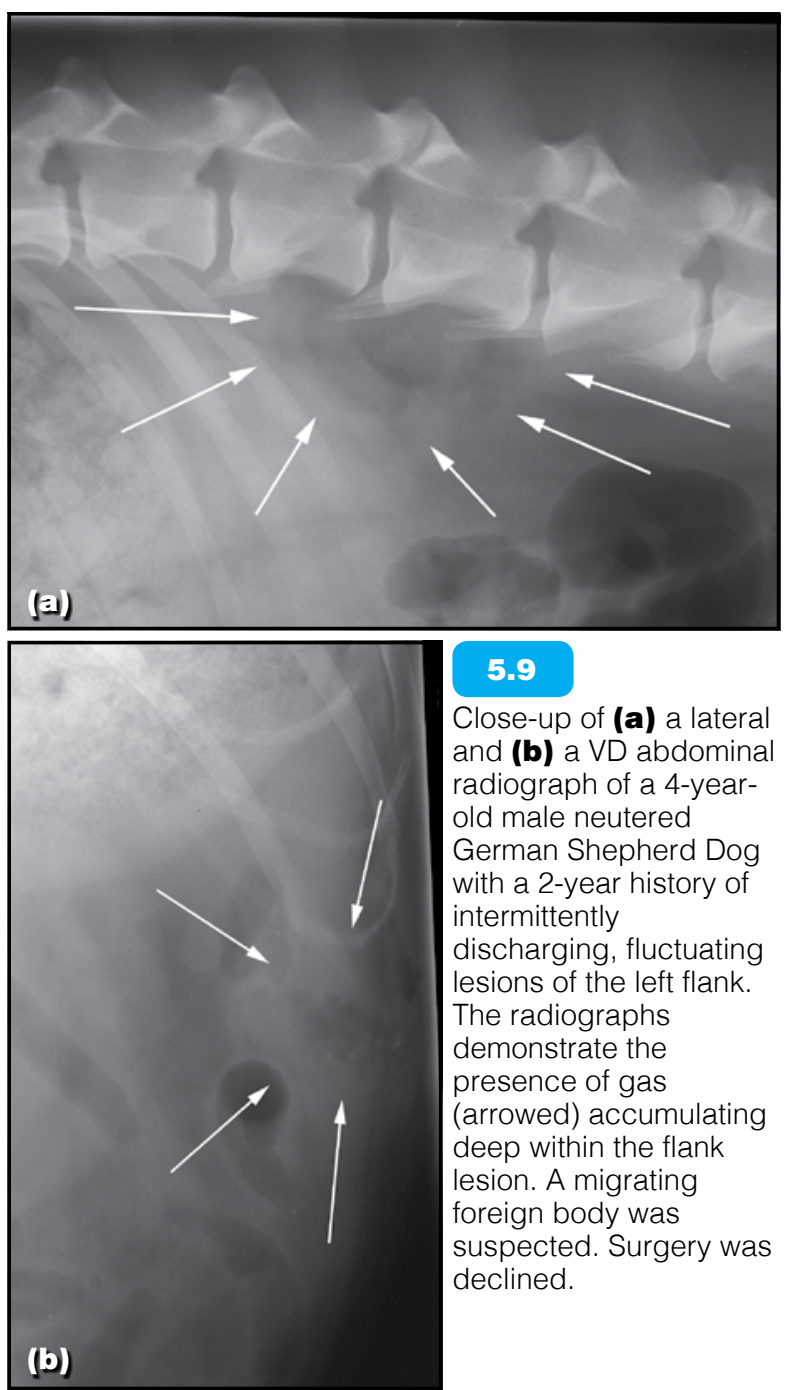

5.9

Close-up of (a) a lateral and (b) a VD abdominal radiograph of a 4 -yearold male neutered German Shepherd Dog with a 2-year history of intermittently discharging, fluctuating lesions of the left flank. The radiographs demonstrate the presence of gas (arrowed) accumulating deep within the flank lesion. A migrating foreign body was suspected. Surgery was declined.

- Although radiographically visible enlargement of the sublumbar muscles has been described, this is considered difficult to identify

- In chronic cases, an irregular periosteal reaction can be seen on the ventral aspect of neighbouring vertebral bodies (spondylitis). The area from T13 to L4 is commonly affected (Figure 5.10). Care must be taken not to confuse spondylitis

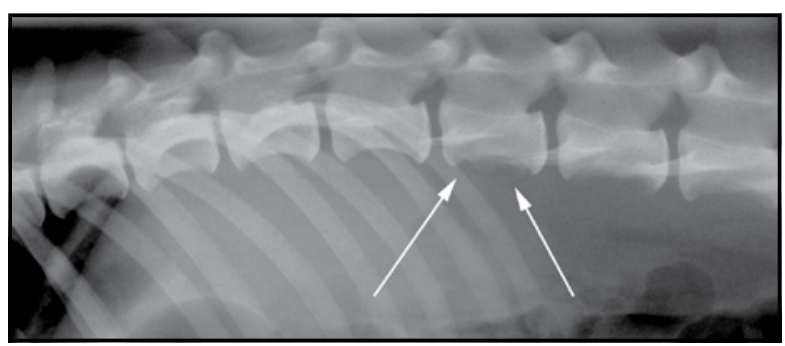

5.10 Close-up of a lateral abdominal radiograph of a 2-year-old male English Cocker Spaniel showing a subtle periosteal reaction arising from the ventral aspect of the second lumbar vertebra. A retroperitoneal abscess was confirmed and surgically debrided following advanced imaging but no foreign body was found with the normal roughening of the ventral aspects of the bodies of the third and fourth lumbar vertebrae associated with the diaphragmatic attachments

- If migration started within the thorax at the level of the peripheral attachments of the diaphragm to the pleura, a periosteal reaction might be seen along the ribs, as well as pleural effusion, pleural thickening and/or focal abscesses. These are infrequent findings.

- Associated discospondylitis may be harder to detect on plain radiographs during the early stages of the disease. Advanced imaging can be more helpful in this situation, as well as for detecting involvement of the epidural space (spinal epidural empyema).

- In extensive cases, and when CT or MRI are not available, sinography (fistulography) can be helpful. This technique is useful if foreign material is demonstrated within the sinus and/or there is an unexpected extension of the sinus tract or communication with another organ cavity (fistula). In such cases, the surgical approach and the extension of the surgical field will be significantly influenced by the results of the contrast study. However, false-negative results are possible if the foreign body remains undetected or the entire length of the sinus is not demonstrated. Falsepositive results are also possible if filling defects interpreted as foreign bodies actually represent tissue fragments, blood clots or focal accumulation of exudate. Therefore, this technique can be misleading in the management of a case and the use of advanced imaging is recommended where available.

- If ultrasonography and sinography are to be used in the same patient, plain radiography and ultrasonography should precede sinography to avoid creating artefacts.

\section{Ultrasonographic findings}

- Ultrasonography is useful in determining whether the retroperitoneal radiographic changes are associated with fluid accumulation, abscessation and/or a parenchymal lesion.

- However, the full extent of the pathology is difficult to determine in severely affected patients, especially if the sublumbar and epaxial musculature is affected, as this area is not routinely investigated when performing a retroperitoneal ultrasound examination. The presence of a focal swelling or a discharging sinus should prompt a more extensive ultrasonographic investigation.

- Ultrasonography has been advocated as a useful tool for the detection of shadowing created by foreign bodies. However, shadowing is not a constant feature, especially with decomposed or very small foreign bodies.

- Grass seeds have a characteristic double or triple spindle shape on ultrasonography and fluid may accumulate around them. 
- Possible explanations for the lack of identification of a foreign body by ultrasonography include:

- Highly cellular fluid around the foreign body

- Complex cellular or gas echoes within an abscess

- Dense fibrous tissue surrounding the foreign body

- Gas trapped within the sinus tract or surrounding the foreign body.

- In some cases, the draining tract can be followed ultrasonographically to the foreign body. Ultrasonography can also be used to guide the retrieval of superficial foreign bodies from within the discharging sinus using forceps or to guide the surgeon to the foreign body, either by positioning a needle close to the foreign body or through intraoperative ultrasonography.

\section{Advanced imaging findings}

In cases of retroperitonitis, surgical exploration with extensive flushing and debridement of abnormal tissue is recommended, followed by antibiotic therapy determined by the results of appropriate culture and sensitivity tests. Advanced imaging plays a fundamental role in determining the extent of the process, especially as both sides of the retroperitoneum may be affected without necessarily showing discharging sinuses on both flanks.

The speed of CT makes it an appealing technique. Reconstructions can be obtained in any plane when using modern systems and software, and this is also useful. Bone detail is exquisite.

However, overall MRI is a superior technique in this situation because of the true multiplanar characteristics of this modality and the array of sequences available. The ability to evaluate the extent of the abscessation, accompanying soft tissue changes and involvement of the vertebral column and epidural space makes this technique attractive (Figure 5.11).

The advantages of some of the sequences available are discussed above. In general, STIR images are useful with low-field MRI systems and are extremely helpful in this clinical scenario (Figure 5.12). Pre- and post-contrast T1-weighted images offer similar information as well as better anatomical detail.

With either CT or MRI the following should be looked for:

- Evidence of retroperitoneal fluid accumulation

- Involvement of neighbouring structures, mainly the paralumbar muscles, vertebrae, the intervertebral discs and the epidural space, as well as the presence of a discharging sinus.

However, a good alignment of the slices with the sinus tract is difficult to achieve and this may be responsible for the lack of identification of the whole length of the tract on CT or MRI images.

Overall, it is very difficult to demonstrate the presence of foreign bodies, especially if they are small.
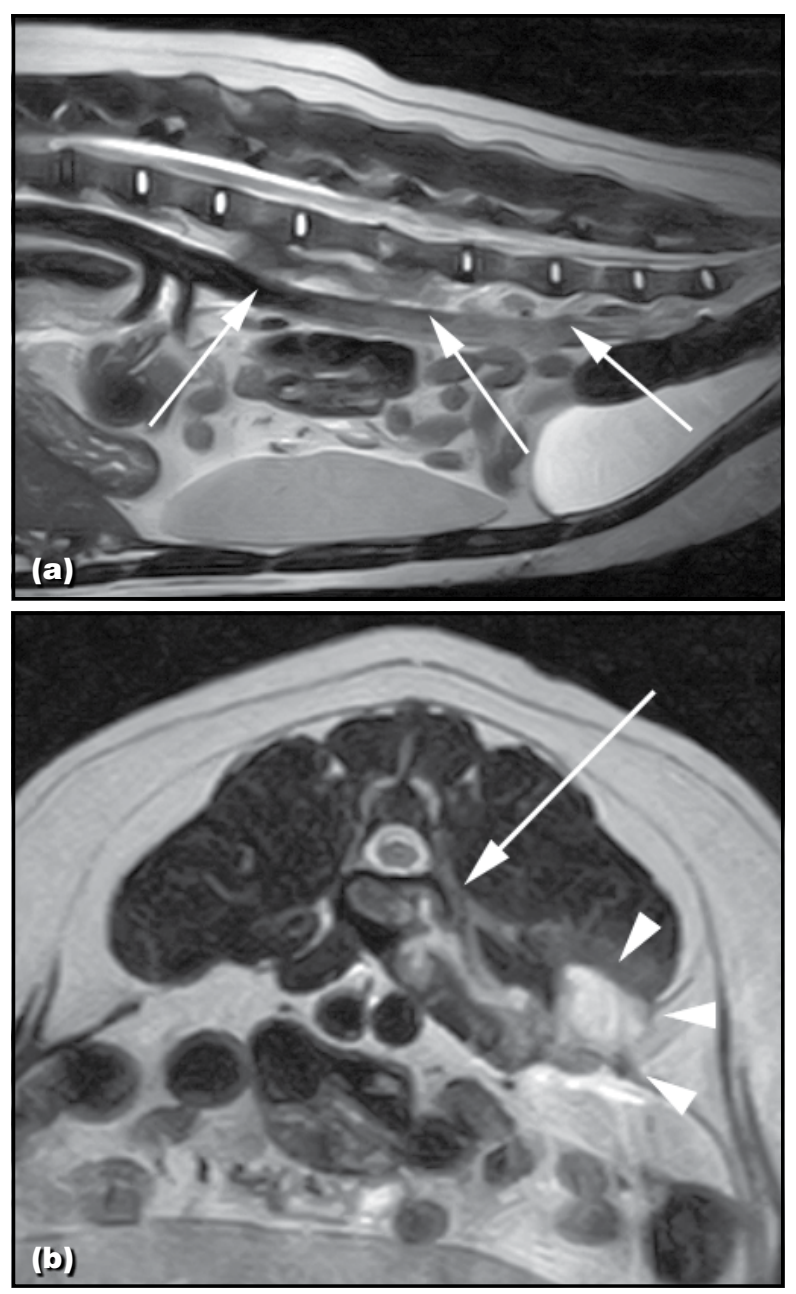

5.11 (a) Sagittal lumbar/abdominal and

(b) transverse T2-weighted MR images of a 2-year-old Labrador Retriever bitch presented for back pain of 6 weeks' duration. The images show not only the presence of retroperitoneal changes compatible with abscessation (arrowed in a) but also the active involvement of the L3-L4 intervertebral disc space (arrowed in b) in the inflammatory process, extending from a large abscess within the paraspinal muscles (arrowheads). Part of a grass seed was retrieved at surgery.

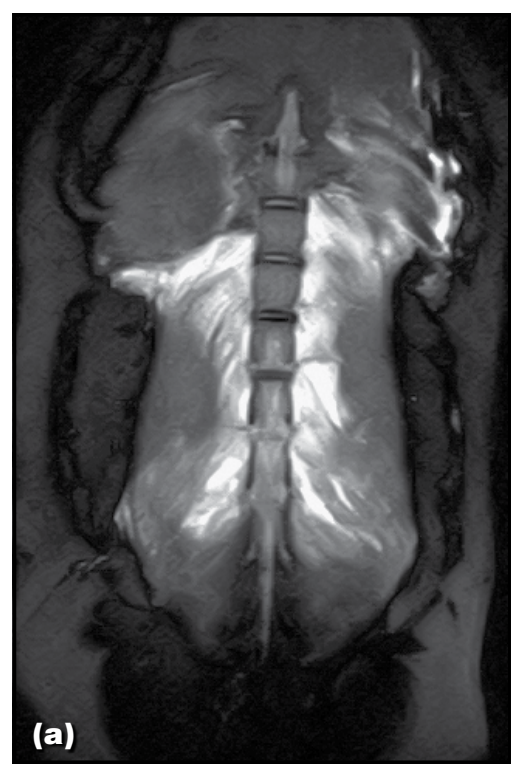

(a) STIR image in the dorsal plane at the level of the paravertebral muscles of the dog in Figure 5.5. This image shows extensive hyperintense regions within the muscle, which were used to direct the surgeon during the cleaning and debriding procedure. (continues) 


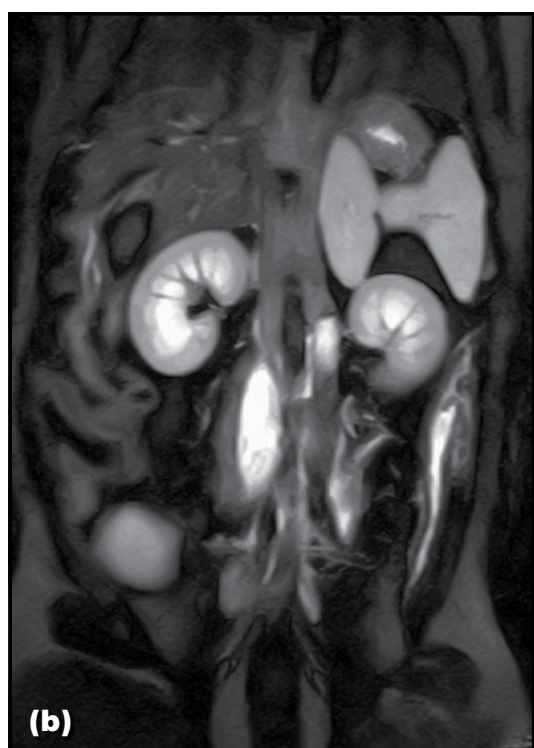

5.12 (continued) (b) STIR image in the dorsal plane at the level of the retroperitoneum of the dog in Figure 5.5. This image shows extensive hyperintense regions within the muscle, which were used to direct the surgeon during the cleaning and debriding procedure.

\section{Air}

Air in the retroperitoneum is most commonly an extension of pneumomediastinum or a result of trauma. A small amount of gas within an abscess or due to focal infection by gas-producing organisms should be differentiated from extensive pneumoretroperitoneum. Depending on the underlying cause, concurrent pneumoperitoneum may be present. Retroperitoneal air can eventually reach the peritoneal cavity.

Plain radiography is particularly useful in patients with pneumoretroperitoneum.

- A decrease in retroperitoneal opacity, together with the clear depiction of retroperitoneal structures, is seen in cases of diffuse pneumoretroperitoneum (Figure 5.13).

- Focal lesions containing gas (for example some abscesses) will also appear radiolucent, but they may not be easy to detect unless they are large and contain fair amounts of gas (see Figure 5.9).

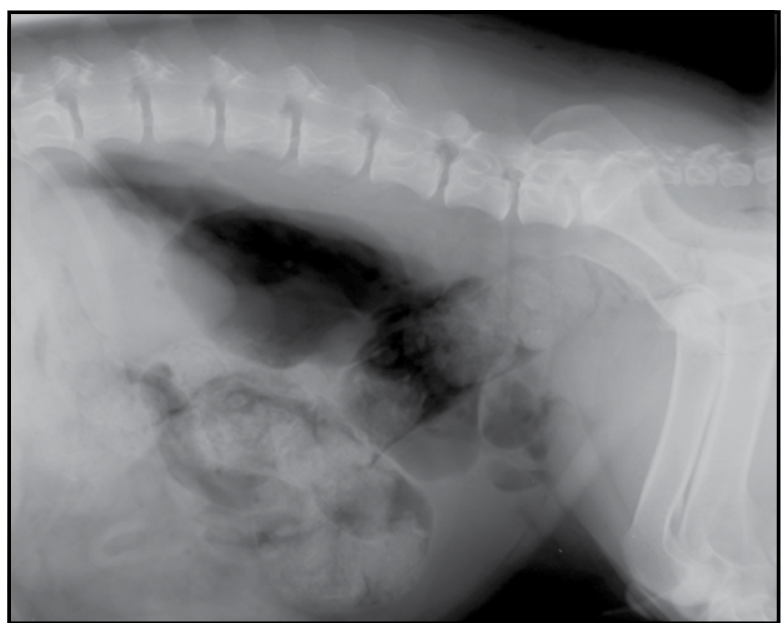

CT may be used to confirm the presence of a small amount of air; it also allows assessment of the mediastinum and other areas connected with it. Ultrasonography and MRI are not considered primary choices in this situation, except in the investigation of focal lesions containing air.

\section{Masses}

Mass lesions are common in the retroperitoneal space. Many are primary or metastatic neoplasms from organs in the retroperitoneal space (e.g. kidney, adrenal gland). Other masses may not have an obvious organ of origin. This is especially true of sarcomas. Haemangiosarcoma is common in the caudal retroperitoneal space without an obvious organ of origin, and may be difficult to identify or characterize owing to surrounding haemorrhage.

Non-neoplastic masses are also possible. The presence of air within the mass should raise the suspicion of an abscess. The presence of mineralization warrants further investigation and a mineralized granuloma or neoplasm should be considered. Mineralized adrenal glands of normal size and shape in cats are usually of no clinical significance. Adrenal gland mineralization is uncommon in dogs as an incidental finding and warrants further studies. Mineralization of an adrenal gland that is abnormal in size or shape in either species requires investigation.

A retroperitoneal mass will displace neighbouring peritoneal structures ventrally and/or to one side. An ovarian mass is peritoneal in origin and, therefore, the neighbouring duodenum or colon will be displaced medially or laterally, but not ventrally. Ventral tipping of the caudal pole of the ipsilateral kidney would be expected.

Periosteal reaction along the mid-ventral margins of the caudal lumbar vertebrae, although not pathognomonic, should raise the suspicion that a caudal abdominal or intrapelvic neoplasm is present with concurrent retroperitoneal extension (Figure 5.14). This periosteal reaction should be distinguished from spondylosis deformans, which affects the vertebral endplates. Spondylitis secondary to foreign body migration will usually affect more cranial lumbar vertebrae (see above). 

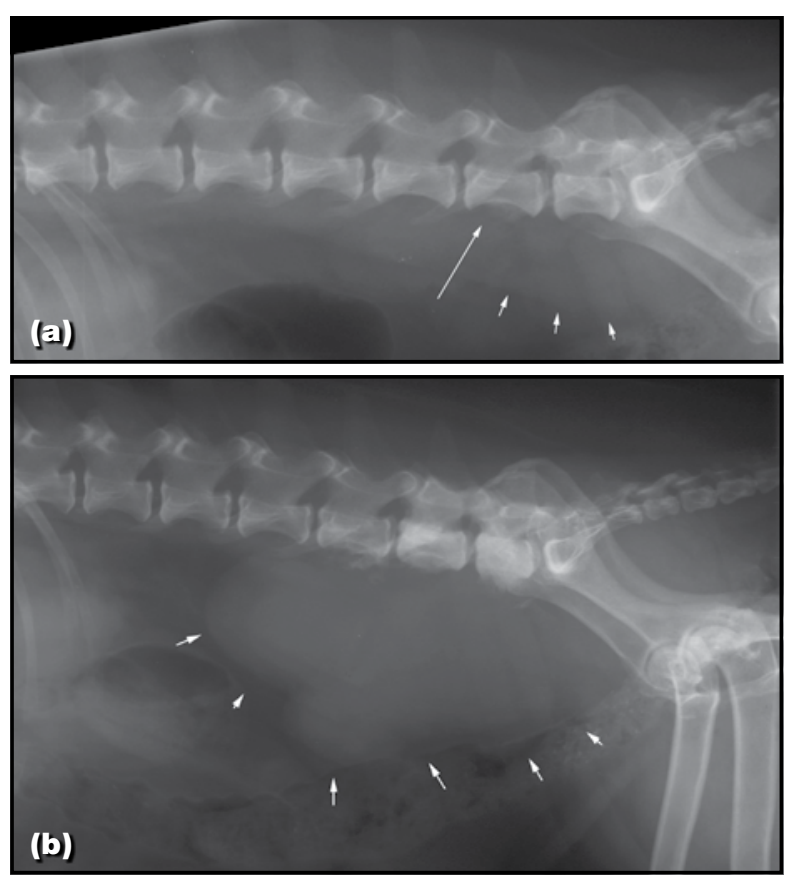

5.14 Close-ups of lateral abdominal radiographs of a 9-year-old male neutered crossbreed dog with adenocarcinoma of the anal glands. An 8-month interval occurred between (a) the first and (b) the second radiograph. (a) Early enlargement of the medial iliac lymph nodes (short arrows) and periosteal reaction of the sixth lumbar vertebra (long arrow) are evident. (b) Dramatic progression is seen with massive enlargement of the lymph nodes (arrowed)

Although use of radiography and ultrasonography can be sufficient to fully investigate some retroperitoneal masses, those patients with very large masses, or where the lesions, regardless of their size, are very close to critical structures such as the aorta, the caudal vena cava or the neighbouring vertebrae and vertebral canal, will benefit from more advanced imaging.

\section{References and further reading}

Armbrust LJ, Biller DS, Radlinsky MG et al. (2003) Ultrasonographic diagnosis of foreign bodies associated with chronic draining tracts and abscesses in dogs. Veterinary Radiology and Ultrasound 44, $66-70$

Brennan KE and Ihrke PJ (1983) Grass awn migration in dogs and cats: a retrospective study of 182 cases. Journal of the American Veterinary Medical Association 182, 1201-1204

Della Santa D, Rossi F, Carlucci F, Vignoli M and Kircher P (2008) Ultrasound guided retrieval of plant awns. Veterinary Radiology and Ultrasound 49, 484-486

Frendin J, Funkquist B, Hansson K, Lonnemark M and Carlsten J (1999) Diagnostic imaging of foreign body reactions in dogs with diffuse back pain. Journal of Small Animal Practice 40, 278-285

Frendin J, Greko C, Hellmen E, Iwarsson M, Gunarsson A and Chryssantou E (1994) Thoracic and abdominal wall swellings in dogs caused by foreign bodies. Journal of Small Animal Practice 35, 499-508

Gnudi G, Volta A, Bonazzi M, Gazzola M and Bertoni G (2005) Ultrasonographic features of grass awn migration in the dog. Veterinary Radiology and Ultrasound 46, 423-426

Heusquin J, Burton CA and Llabres-Diaz FJ (2007) Outcome of sublumbar abscesses following surgical management guided by diagnostic imaging: study of 14 dogs. Proceedings of the BSAVA Congress 2007, p. 528

Holloway A and O' Brien R (in press) Perirenal effusion in dogs and cats with acute renal failure. Veterinary Radiology and Ultrasound

Johnston DE and Christie BA (1990a) The retroperitoneum in dogs: anatomy and clinical significance. Compendium on Continuing Education for the Practicing Veterinarian 12, 1027-1033

Johnston DE and Christie BA (1990b) The retroperitoneum in dogs: retroperitoneal infections. Compendium on Continuing Education for the Practicing Veterinarian 12, 1035-1045

Lamb CR, White RN and McEvoy FJ (1994) Sinography in the investigation of draining tracts in small animals: retrospective review of 25 cases. Veterinary Surgery 23, 129-134

Liptak JM, Dernell WS, Ehrhart EJ et al. (2004) Retroperitoneal sarcomas in dogs: 14 cases (1992-2002). Journal of the American Veterinary Medical Association 224, 1471-1477

McEvoy FJ, Lamb CR and White RN (1993) An application of sinography in small animal practice. Veterinary Record 132, 183-185

Penninck D and Mitchell SL (2003) Ultrasonographic detection of ingested and perforating wooden foreign bodies in four dogs. Journal of the American Veterinary Medical Association 223, 206-209

Roush JK, Bjorling DE and Lord P (1990) Diseases of the retroperitoneal space in the dog and cat. Journal of the American Animal Hospital Association 26, 47-54

Saunders WB and Tobias KM (2003) Pneumoperitoneum in dogs and cats: 39 cases (1983-2002). Journal of the American Veterinary Medical Association 223, 462-468

Schermerhorn T, McNamara PS, Dykes NL and Toll J (1998) Cullen's sign and haemoglobinuria as presenting signs of retroperitoneal haemorrhage in a dog. Journal of Small Animal Practice 39, 490-494 